\title{
Uso, Necessidade e Conservação de Próteses Dentárias em Idosos Residentes em Instituições Públicas de Longa Permanência
}

\author{
Use, Necessity and Preservation of Dental Prostheses in Elderly Residents in \\ Public Institutions of Long Stay
}

\author{
Paulo José de Lima' \\ Camila Agra ${ }^{2}$ \\ Lívia Maria Lopes ${ }^{2}$ \\ Cátia Maria Guerra Fonseca ${ }^{3}$
}

\section{RESUMO}

Objetivo: Conhecer o uso, as condições e a necessidade de prótese dentária entre idosos residentes em instituições públicas de longa permanência (IPLP) para idosos do Município do Recife. Metodologia: Para composição da amostra deste estudo, de um universo de 60 idosos, residentes em IPLP da Cidade do Recife, participaram da amostra 47 idosos, que se enquadraram nos critérios de inclusão, ou seja, se mostraram receptivos a participar da pesquisa e no máximo apresentavam comprometimento cognitivo que não prejudicasse as funções de linguagem e memória. Resultados: Na amostra a idade dos pesquisados variou de 52 a 87 anos, com uma média de 65,00 anos, sendo que $34 \%$ eram do sexo masculino e $66 \%$ do sexo feminino. O estudo revelou que $70,2 \%$ usavam prótese no arco superior e $48,9 \%$ no arco inferior. Apesar de 72,7\% informarem o uso de diferentes meios para a higiene oral, a presença de biofilme foi detectada em $74,4 \%$ das próteses. Grande parte dos idosos $(66,0 \%)$ necessitava de substituição das próteses, consideradas inadequadas ou para reabilitação. Conclusão: Conclui-se que o idoso não consegue perceber as condições precárias da prótese, existindo a necessidade de maior atenção à saúde bucal do idoso institucionalizado e a elaboração de programas preventivos e educativos voltados para essa parcela da população.

\section{DESCRITORES}

Idoso. Prótese Dentária. Instituição de Longa Permanência para Idosos.

\begin{abstract}
Objective: To know the use, conditions and the need for dentures among elderly residents in public long-stay institutions for the elderly (PNHs) in the city of Recife. Methods: For the sample composition of this study, of a population of 60 seniors, 47 seniors participated in the sample that met the selection criteria, ie were receptive to participate and at most had cognitive impairment that did not impair the functions of language and memory. Results: In the sample of respondents, age ranged from 52 to 87 years, with an average of 65 years, of which $34.0 \%$ were males and $66.0 \%$ were female. The study revealed that $70.2 \%$ used prosthesis in the maxillary arch and $48.9 \%$ in the lower arch. Although $72.7 \%$ reported the use of different means for oral hygiene, the presence of biofilm was detected in $74.4 \%$ of the prostheses. The majority $(66.0 \%)$ of the elderly needed replacement of prostheses, considered inadequate or for rehabilitation. Conclusion: The elderly cannot understand the precarious conditions of the prosthesis and there is a need for greater attention to the oral health of institutionalized elders and the development of preventive and educational programs for this population.
\end{abstract}

\section{DESCRIPTORS}

Aged. Dental Prosthesis. Homes for the Aged.

${ }^{1}$ Cirurgião-dentista, Universidade Federal de Pernambuco - UFPE, Recife, Pernambuco, Brasil.

${ }^{2}$ Doutoranda do Programa de Pós-Graduação em Odontologia (Clínica Integrada), Universidade Federal de Pernambuco - UFPE, Recife, Pernambuco, Brasil.

${ }^{3}$ Professora do Departamento de Prótese e Cirurgia Buco-Facial da Universidade Federal de Pernambuco - UFPE, Recife, Pernambuco, Brasil. 
E nvelhecer é um processo natural, que caracteriza uma etapa na vida do homem e ocorre por mudanças físicas, psicológicas e sociais ${ }^{1}$. A institucionalização pode trazer como consequência a perda, aos poucos, do relacionamento com os familiares e amigos e este sentimento de abandono contribui para a deterioração da saúde geral do paciente, com implicação direta na cavidade bucal ${ }^{2,3}$.

Sabe-se que as condições sociais dos indivíduos bem como a prática odontológica hegemônica, que tem nas extrações dentárias a solução para o alívio da dor em populações de baixo nível socioeconômico, contribuem para o edentulismo elevado no país, especialmente, na faixa etária de 65 a 74 anos $^{4}$.

A prevalência de uso e necessidade de prótese dentária, de acordo com a Pesquisa Nacional de Saúde Bucal de $2010^{5}$, foi de $78,2 \%$ e $68,7 \%$, respectivamente. A Região Nordeste foi a que apresentou menor prevalência de uso (71, 3\%) e maior de necessidade de prótese dentária $(82,9 \%)^{6}$. Assim sendo, pode-se dizer que grande parte dos idosos brasileiros ou são usuários de próteses ou necessitam utilizá-las ${ }^{7,8}$.

A saúde bucal de idosos residentes em instituições de longa permanência (ILP) parece não diferir deste perfil. Gaião, Almeida e Heukelbach ${ }^{9}$ observaram um CPO-D de 29,73, entre idosos institucionalizados no Ceará, com o componente perdido representando o maior valor. Outros estudos ${ }^{8,9}$ demonstraram a precariedade da saúde bucal de idosos em IPLP, representada por higiene oral deficiente, alta experiência de cárie, perda dentária, além de presença de bolsa. Poucos estudos ${ }^{10}$, entretanto, avaliaram as condições de con- servação de próteses dentárias pelos idosos institucionalizados.

Considerando o envelhecimento populacional e as necessidades de reabilitação protética para esta população ${ }^{11}$, este trabalho teve como objetivo investigar as condições de uso, necessidade e conservação de prótese dentária entre indivíduos residentes em IPLP pública, no Município de Recife/PE.

\section{METODOLOGIA}

Trata-se de um estudo de corte-transversal e analítico, desenvolvido dentro do projeto de extensão universitária Promoção de Saúde Bucal para Idosos Residentes em Instituições Públicas de Longa Permanência, na Cidade do Recife. O estudo recebeu a aprovação do Comitê de Ética da Universidade Federal de Pernambuco, com o parecer de número 152.113. Um termo de consentimento livre e esclarecido foi assinado por cada participante, antes de todos os procedimentos de investigação.

A pesquisa foi realizada no Abrigo lêda Lucena, Lar Batista de Carvalho e Lar de Maria, localizadas nos Distritos Sanitários IV e VI. Todas as instituições estão vinculadas a Secretaria de Saúde do Município do Recife/ PE. Para participar do estudo, os residentes não poderiam ter comprometimento cognitivo que prejudicassem as funções de linguagem e memória. Ao todo, 60 idosos residiam nessas instituições, no entanto, apenas 47 , aceitaram participar do estudo ou enquadraram-se no critério de inclusão.

A coleta de dados ocorreu a partir de uma entrevista estruturada e de exame clínico, registrados em ficha clínica especifica. Os 
exames clínicos foram realizados por cinco examinadores, previamente calibrados $(\mathrm{k}=$ $0,72)$, sob condições idênticas. Para aqueles que utilizavam próteses, essas foram examinadas quanto à presença de biofilme, tártaro, fraturas, desgastes dentários, perfurações, consertos, tempo de uso e perguntado como era realizada a higienização da mesma. As variáveis dependentes foram as dimensões de dor conforto, restrição alimentar, desempenho e aparência e as variáveis independentes foram: condições sociodemográficas - faixa etária e sexo; as variáveis relacionadas com as condições de saúde bucal - necessidade declarada de prótese dentaria.

Os dados foram analisados descritivamente de forma uni e bivariada por meio de distribuições absolutas, percentuais para as variáveis categóricas e das medidas estatísticas: média, desvio padrão e mediana para variáveis numéricas. $\mathrm{O}$ teste exato de Fisher foi utilizado para testar a hipótese de associação significativa entre as variáveis categóricas e o teste de Mann-Whitney para a comparação entre os sexos em relação as variáveis numéricas. A margem de erro utilizada nas decisões dos testes estatísticos foi de $5 \%$. O programa estatístico utilizado para digitação dos dados e obtenção dos cálculos estatísticos foi o SPSS (IBM SPSS Statistics 21).

\section{RESULTADOS}

A idade dos pacientes variou entre 52 a 87 anos, com média de $65,70 \pm 6,6$ anos, $55,3 \%$ tinham de 52 a 65 anos, os demais 66 anos ou mais. Indivíduos do sexo feminino representaram $66 \%$ dos participantes.

A Tabela 1 apresenta os resultados relativos à presença de dentes e uso de próteses por arco. Menos da metade (48,9\%) dos participantes tinha dentes no arco superior, enquanto, $63,8 \%$ tinham dentes no arco inferior. Quanto ao uso de prótese, $29,8 \%$ dos participantes não utilizavam.

A maior parte fazia uso de prótese no arco superior $(70,2 \%)$, sendo que $51,1 \%$ utilizava prótese total e $19,1 \%$ prótese parcial removível, apenas $6,4 \%$ delas foram avaliadas como em boas condições. Entre os que utilizavam prótese no arco inferior, $25,5 \%$ tinham prótese total e $23,4 \%$ prótese parcial

Tabela 1. Avaliação da presença de dentes e uso de próteses utilizada por arco

\begin{tabular}{l|c|c|c|c|c|c}
\hline \multirow{2}{*}{ Variável } & \multicolumn{6}{c}{ Arco } \\
\cline { 2 - 8 } & \multicolumn{2}{|c|}{ Superior } & \multicolumn{2}{c}{ Inferior } & \multicolumn{2}{c}{$\begin{array}{c}\text { Independente do } \\
\text { arco }\end{array}$} \\
\cline { 2 - 8 } & $\mathrm{n}=47$ & $\%(100)$ & $\mathrm{n}=47$ & $\%(100)$ & $\mathrm{n}=47$ & $\%(100)$ \\
\hline Tem dentes & & & & & & \\
\hline Sim & 23 & 48,9 & 30 & 63,8 & 33 & 70,2 \\
\hline Não & 24 & 51,1 & 17 & 36,2 & 14 & 29,8 \\
\hline Uso prótese & & & & & & \\
\hline Sim & 33 & 70,2 & 23 & 48,9 & 33 & 70,2 \\
\hline Não & 14 & 29,8 & 24 & 51,1 & 14 & 29,8 \\
\hline
\end{tabular}


Tabela 2. Tipos e condição das próteses utilizada por arco

\begin{tabular}{|c|c|c|c|c|}
\hline \multirow{3}{*}{ Variável } & \multicolumn{4}{|c|}{ Arco } \\
\hline & \multicolumn{2}{|c|}{ Superior } & \multicolumn{2}{|c|}{ Inferior } \\
\hline & $\mathrm{n}=47$ & $\%(100)$ & $\mathrm{n}=47$ & $\%(100)$ \\
\hline \multicolumn{5}{|l|}{ Tipo de prótese que utiliza } \\
\hline Prótese total & 24 & 51,1 & 12 & 25,5 \\
\hline Prótese parcial removível & 09 & 19,1 & 11 & 23,4 \\
\hline Não utiliza prótese no arco & - & - & 10 & 21,3 \\
\hline Não utiliza próteses & 14 & 29,8 & 14 & 29,8 \\
\hline \multicolumn{5}{|l|}{ Boa condição da prótese } \\
\hline Sim & 03 & 6,4 & - & - \\
\hline Não & 25 & 53,2 & 20 & 42,6 \\
\hline Não utiliza próteses & 14 & 29,8 & 24 & 51,1 \\
\hline Não informado & 05 & 10,6 & 03 & 6,4 \\
\hline \multicolumn{5}{|l|}{ Biofilme na prótese } \\
\hline Sim & 20 & 42,6 & 15 & 31,9 \\
\hline Não & 08 & 17,0 & 05 & 10,6 \\
\hline Não utiliza prótese & 14 & 29,8 & 24 & 51,1 \\
\hline Não informado & 05 & 10,6 & 03 & 6,4 \\
\hline \multicolumn{5}{|l|}{ Desgaste na prótese } \\
\hline Sim & 14 & 29,8 & 7 & 14,9 \\
\hline Não & 14 & 29,8 & 13 & 27,7 \\
\hline Não utiliza prótese & 14 & 29,8 & 24 & 51,1 \\
\hline Não informado & 05 & 10,6 & 03 & 6,4 \\
\hline \multicolumn{5}{|l|}{ Tártaro na prótese } \\
\hline Sim & 18 & 38,3 & 14 & 29,8 \\
\hline Não & 10 & 21,3 & 06 & 12,8 \\
\hline Não utiliza prótese & 14 & 29,8 & 24 & 51,1 \\
\hline Não informado & 05 & 10,6 & 03 & 6,4 \\
\hline \multicolumn{5}{|l|}{ Fratura na prótese } \\
\hline Sim & 07 & 14,9 & 01 & 2,1 \\
\hline Não & 21 & 44,7 & 19 & 40,4 \\
\hline Não utiliza prótese & 14 & 29,8 & 24 & 51,1 \\
\hline Não informado & 05 & 10,6 & 03 & 6,4 \\
\hline \multicolumn{5}{|l|}{ Já foi realizado conserto } \\
\hline Sim & 05 & 10,6 & 02 & 4,3 \\
\hline Não & 23 & 48,9 & 18 & 38,3 \\
\hline Não utiliza prótese & 14 & 29,8 & 24 & 51,1 \\
\hline Não informado & 05 & 10,6 & 03 & 6,4 \\
\hline
\end{tabular}


removível, nenhuma considerada em boas condições.

A presença de biofilme foi verificada em $42,6 \%$ das próteses do arco superior e $31,9 \%$ das do arco inferior. A presença de desgaste foi verificada em $29,9 \%$ no arco superior e $14,9 \%$ no arco inferior; o tártaro ocorreu em $38,3 \%$ no arco superior e $29,8 \%$ no arco inferior. As fraturas foram registradas em $14,9 \%$ no arco superior e em $2,1 \%$ no arco inferior. A realização de conserto tinha ocorrido em $10,6 \%$ das próteses do arco superior e em $4,3 \%$ do arco inferior (Tabela 2 ).

Para $66 \%$ dos pacientes detectou-se a necessidade de próteses seja pela ausência, pelo tempo de uso superior a 5 anos ou porque as próteses tinham desgaste ou fratura. Entre os pacientes que utilizavam próteses, $40,4 \%$ responderam que mastigavam bem (Tabela 2). Em relação à forma de higienização da prótese, 48,9\% responderam que limpavam a prótese com o auxílio de escova associada a pasta de dentes, ao passo que $10,6 \%$ acrescentavam hipoclorito de sódio. Outras formas citadas tiveram percentuais que variaram de $2,1 \%$ a $4,3 \%$, conforme resultados apresentados na Tabela 3.

A associação entre o uso e a necessidade de próteses (Tabela 4) demonstrou que $72,7 \%$ dos indivíduos que utilizavam prótese precisavam substituí-la $(p>0,05)$.

Indivíduos pertencentes ao sexo feminino mostraram maior tempo relacionado à perda dentária, com média de 27,42 anos, com diferença estatisticamente significativa, quando comparada ao tempo de perda do sexo masculino (Tabela 5). O tempo médio de uso da prótese atual mostrou ser superior a cinco anos, entre os participantes do estudo.

Tabela 3. Avaliação da necessidade de prótese (s), de acordo com a avaliação da mastigação e a forma de higienização da prótese

\begin{tabular}{l|c|c}
\hline \multicolumn{1}{c|}{ Variável } & $\mathrm{n}$ & $\%$ \\
\hline Necessidade de prótese & & \\
\hline Sim & 31 & 66,0 \\
\hline Não & 16 & 34 \\
\hline Mastiga bem com as próteses & & \\
\hline Sim & 19 & 40,4 \\
\hline Não & 14 & 29,8 \\
\hline Não utilizava próteses & 14 & 29,8 \\
\hline Forma de higienização da prótese & & \\
\hline Escova + pasta dental & 23 & 48,9 \\
\hline Água sanitária & 02 & 4,3 \\
\hline Sabão neutro + água sanitária & 01 & 2,1 \\
\hline Escova + pasta dental + água sanitária & 05 & 10,6 \\
\hline Escova + pasta dental + detergente neutro & 01 & 2,1 \\
\hline Detergente neutro + água sanitária & 01 & 2,1 \\
\hline Não usava próteses & 14 & 29,8 \\
\hline
\end{tabular}


Tabela 4. Avaliação da necessidade de prótese

\begin{tabular}{l|c|c|c|c|c|c|c}
\hline & \multicolumn{4}{|c|}{ Necessidade de prótese } & \multicolumn{2}{c|}{} & \\
\hline Uso de prótese & \multicolumn{2}{|c|}{$\operatorname{Sim}$} & \multicolumn{2}{c|}{ Não } & \multicolumn{2}{c|}{ TOTAL } & Valor de $\mathrm{p}$ \\
\hline & $\mathrm{n}$ & $\%$ & $\mathrm{n}$ & $\%$ & $\mathrm{n}$ & $\%$ & \\
\hline Sim & 24 & 72,7 & 09 & 27,3 & 33 & 100,0 & $\mathrm{p}^{(1)}=0,182$ \\
\hline Não & 07 & 50,0 & 07 & 50,0 & 14 & 100,0 & \\
\hline Grupo Total & 31 & 66,0 & 16 & 34,0 & 47 & 100,0 & \\
\hline
\end{tabular}

(1) Através do teste Exato de Fisher.

Tabela 5. Tempo de desdentado na maxila, tempo de desdentado na mandíbula e tempo de uso da prótese atual no grupo total e segundo o sexo

\begin{tabular}{|c|c|c|c|c|}
\hline \multirow[b]{2}{*}{ Tempo analisado } & \multicolumn{2}{|c|}{ Sexo } & \multirow[b]{2}{*}{ Grupo total } & \multirow[b]{3}{*}{ Valor de $p$} \\
\hline & Masculino & Feminino & & \\
\hline & $\begin{array}{c}\text { Média } \pm \text { DP }(\mathrm{Me}- \\
\text { diana) }\end{array}$ & $\begin{array}{l}\text { Média } \pm \text { DP } \\
\text { (Mediana) }\end{array}$ & $\begin{array}{c}\text { Média } \pm \text { DP (me- } \\
\text { diana) }\end{array}$ & \\
\hline De desdentado maxilar & $\begin{array}{c}14,67 \pm 12,21 \\
(10,00)\end{array}$ & $\begin{array}{c}27,42 \pm 13,13 \\
(21,00)\end{array}$ & $\begin{array}{c}22,48 \pm 14,07 \\
(20,00)\end{array}$ & $p^{(1)}=0009^{*}$ \\
\hline De desdentado mandibular & $\begin{array}{c}16,00 \pm 11,29 \\
(11,00)\end{array}$ & $\begin{array}{c}19,66 \pm 11,98 \\
(20,00)\end{array}$ & $\begin{array}{c}18,13 \pm 11,60 \\
(17,50)\end{array}$ & $\mathrm{p}^{(1)}=0,374$ \\
\hline Da prótese atual & $\begin{array}{c}7,80 \pm 8,51 \\
(5,00)\end{array}$ & $\begin{array}{c}6,69 \pm 4,08 \\
(7,00)\end{array}$ & $\begin{array}{c}6,99 \pm 5,44 \\
(6,50)\end{array}$ & $\mathrm{p}^{(1)}=0,614$ \\
\hline
\end{tabular}

'Diferença significativa ao nível de 5,0\%.

(1) Através do teste de Mann-Whitney.

\section{DISCUSSÃO}

A institucionalização da população idosa cresce proporcionalmente à ascensão da expectativa de vida, o que tem aumentado a necessidade de atendimento e de cuidados em saúde ${ }^{12}$. Neste estudo foi verificada que $66 \%$ dos moradores das IPLP eram mulheres. A média de idade dos idosos foi de 65,7 anos, inferior à de outros estudos que investigaram a mesma população ${ }^{13-17}$, cujas médias variaram entre 76 e 81 anos.

No Brasil, o edentulismo constitui um problema de saúde pública. De acordo com o último levantamento de saúde bucal, apenas $23,5 \%$ da população na faixa etária de 65 a 74 anos não usava algum tipo de prótese dentária superior, sendo maior o percentual na Região Nordeste (31,4\%). Entre os idosos participantes do estudo, observou-se que $70,2 \%$ utilizavam algum tipo de prótese superior. Essa discrepância pode ser explicada pela natureza das instituições componentes do estudo, considerados espaços para o acolhimento de idosos, com referência familiar ou abandonados, sem condições de se manter, ou seja, um grupo selecionado de indivíduos provavelmente submetidos, ao longo da vida, a um acesso limitado a serviços voltados para cuidados odontológicos.

Vários autores identificaram que além das causas sociais para o edentulismo está 
a organização dos serviços de saúde bucal. Estes, ao longo da história, eram pensados exclusivamente como assistência cirúrgico-restauradora, o que colocava a extração dentária como uma das principais opções de tratamento, frente a impossibilidade econômica para a realização de tratamentos mais conservadores. A extração dentária seria, então, vista como um processo comum ao curso da vida ${ }^{18,19}$.

O estudo de Lewandowiski e Bós ${ }^{13}$ avaliou o uso e a necessidade de prótese em idosos longevos, identificando que 76,3\% deles utilizavam prótese total superior, 39,6\% prótese inferior, enquanto o percentual encontrado no presente estudo foi de $51,1 \%$ de usuários de prótese total superior e $48,9 \%$ utilizavam prótese inferior (Tabela 1). Este estudo avaliou uma população específica, usuária de um serviço multiprofissional de Geriatria e Gerontologia, o que pode ter introduzido um viés de seleção nos resultados. Os usuários de próteses parciais removíveis superiores e inferiores representaram 19,1 e 23,4\% (Tabela 2), respectivamente, como observado em outros estudos, como o de Lewinski et al. ${ }^{16}$

A análise das condições da prótese, quanto ao acúmulo de biofilme e a presença de tártaro, revelou que existia a presença de biofilme em $42,6 \%$ das próteses superiores e $31,9 \%$ das próteses inferiores (Tabela 2 ). Quando estes dados são comparados com a forma relatada de higienização, observou-se que $70,2 \%$ dos usuários de próteses, relataram que executavam algum tipo de higienização, seja pelo uso exclusivo de escova e pasta dental ou associado a outras substâncias (água sanitária, detergente ou sabonete neutro). Esses dados sinalizam uma ineficiência associada ao ato de limpeza da prótese, que pode ser devida a dificuldades motoras ${ }^{10}$ ou desconhecimento de métodos de higienização das próteses pelos idosos e/ou cuidadores.

Quanto as condições da prótese, $44,6 \%$ apresentavam desgaste, $29,8 \%$ em próteses superiores e 14,9\% em inferiores, em $17 \%$ das próteses foram observadas fraturas e 14,8\% já tinham sido submetidas a conserto (Tabela 2). O tempo médio de uso das próteses foi superior a cinco anos, o que pode explicar esses achados. Cardenas e Paraguassu ${ }^{20}$ encontraram uma correlação positiva entre um maior tempo de uso e a piora da qualidade de vida de usuários de próteses mucossuportadas.

Com relação a eficiência mastigatória, $29,8 \%$ dos participantes informaram não mastigar bem com as próteses (Tabela 4). Essa dificuldade pode ser explicada pela diminuição do tônus muscular e da coordenação da musculatura envolvida na mastigação, pela perda da dentição natural e pela má adaptação de próteses dentárias, sendo que estes fatores afetam diretamente a preparação do bolo alimentar e ocasionam maior dificuldade com alimentos duros e fibrosos. Sabe-se que a condição dentária influencia no processo de alimentação, interferindo na função de mastigação, afetando o preparo de o bolo alimentar e, consequentemente, dificultando sua deglutição, situação que muitas vezes ocasiona no idoso desconforto, diminuição da ingesta de alimentos sólidos e perda do prazer em se alimentar. ${ }^{21}$

A necessidade de prótese, considerando usuários e não usuários, esteve presente em $66 \%$ da amostra. Quando se compara o tempo de desdentado em ambos os arcos, o maior tempo foi observado no sexo feminino com a média de 27 anos na maxila e 19 na mandíbula. Esse fato pode ser explicado pela maior procura por mulheres a cuidados e serviços de saúde. 


\section{CONCLUSÃO}

Com base na análise dos dados observados pelo presente estudo, pode-se concluir que existe grande necessidade de prótese entre os idosos residentes nas instituições que participaram da pesquisa, considerando indivíduos que tinham perda dentária e não utilizavam próteses e aqueles que utilizavam

\section{REFERÊNCIAS}

1. Rocha MAS, Lima MLLT.Caracterização dos distúrbios miofuncionais orofaciais de idosos institucionalizados. Geriatria \& Gerontologia. 2010; 1(4):21-26.

2. Freitas MAV, Scheicher ME. Qualidade de vida de idosos institucionalizados. Rev. Bras. Geriatr. Gerontol. 2010; 3(13):395-401.

3. Beloti AM, Schwab BL, Bertipaglia T, Nishimori LE, Molena-Fernandes CA. Avaliação das condições de saúde bucal de idosos institucionalizados em asilos públicos de maringá-pr. Cienc Cuid Saúde. 2011;1(10):96-100.

4. Silva ET, Oliveira RT, Leles CR. O edentulismo no Brasil: epidemiologia, rede assistencial e produção de próteses pelo Sistema Único de Saúde. Tempus, Actas de Saúde Colet. 2015;9(3):121-134

5. BRASIL. Ministério da Saúde. Secretaria de Atenção à Saúde. Departamento de Atenção Básica. Projeto SB Brasil 2010: condições de saúde bucal da população brasileira 2010: resultados principais. Brasília: Ministério da Saúde; 2012.

6. Azevedo JS, Azevedo MS, Oliveira LJC, Correia MB, Demarco FF. Uso e necessidade de prótese dentária em idosos brasileiros segundo a Pesquisa Nacional de Saúde Bucal (SBBrasil 2010): prevalências e fatores associados. 2017; 33(8): e00054016.

7. Roncalli AG. Epidemiologia em saúde bucal coletiva: um caminhar compartilhado. Ciênc Saúde Coletiva.2006;11(1):105-114

8. Furtado DG, Forte FDS, Leite DFBM. Uso e Necessidade de Próteses em Idosos: Reflexos na Qualidade de Vida. Revista Brasileira de Ciências da Saúde. 2011;15(2):183-190.

9. Gaião LR, Almeida MEL, Heukelbach J. Perfil epidemiológico da cárie dentária, doença periodontal, uso e necessidade de prótese em idosos residentes em uma instituição na cidade de Fortaleza, Ceará. Rev Bras Epidemiol. 2005; 8(3):316-23.

10. Ferreira RC, Magalhães CS, Rocha ES, Schwambach CW, Moreira AN. Saúde bucal de idosos residentes em instituições de longa permanência de Belo Horizonte, Minas Gerais, Brasil. Cad. Saúde Pública. 2009; 25(11):2375-2385.

11. Sá IPC, Almeida Júnior LR, Corvino MPF, Sá SPC. Condições de saúde bucal de idosos da instituição de longa permanência Lar Samaritano no município de São Gonçalo-RJ. Ciência \& Saúde Coletiva. 2012;17(5):1259-1265. próteses com estado de conservação precário.

É de extrema importância promover trabalhos de educação e saúde, enfatizando o incentivo da higienização das próteses não somente entre os idosos, mas também para os cuidadores, além de um acompanhamento pelo cirurgião-dentista para identificação de demandas em discussão da saúde bucal e prevenção de alterações bucais.

12. Sá IPC, Sá SPC, Oliveira Filho VC. Avaliação comparativa das condições bucais do idoso: ações para o cuidado e autocuidado. Revista Kairós-Gerontologia. 2019; 22(3): 85-98.

13. Lewandowisk A, Bós, AJG. Estado de saúde bucal e necessidade de prótese dentária em idosos longevos. Rev Assoc Paul Cir Dent. 2014;68(2):155-158.

14. Reis SCGB, Higino MASP, Melo HMDM, Freire MCM Condição de saúde bucal de idosos institucionalizados em Goiânia-GO, 2003. Rev Bras Epidemiol. 2005; 8(1):67-73.

15. Falcão TN, Costa MMA, Fernandes LM, Almeida LFD, Valença AMG. Qualidade de Vida e Condições de Higiene de Próteses Dentárias de Idosos Institucionalizados. Rev Bras de Ciências da Saúde. 2019; 23(1):73-80.

16. Levinski E, Schuc KL, Scortegagna SA, Levinsky E, Zanette F, De Carli JP, Trentin MS. Atenção à Saúde Bucal do Idoso Institucionalizado por meio de Ações de Extensão Universitária. 2017; 36(2):393-408.

17. Braz ESV, Gomes RSLCS. Conhecimento de idosos sobre alimentação saudável em uma instituição de longa permanência em Belém-Pará. 2020; 6(2):8685-8693.

18. Vargas AMD, Paixão HH. Perda dentária e seu significado na qualidade de vida de adultos usuários de serviço público de saúde bucal do Centro de Saúde Boa Vista, em Belo Horizonte. Ciênc Saúde Coletiva. 2005;10(4):1015-1024.

19. Martins AMEBL, Barreto SM, Pordeus IA. Autoavaliação de saúde bucal em idosos: análise com base em modelo multidimensional. Cad Saúde Pública. 2009; 25(2):421-435.

20. Cardenas AMC, Paraguassu EC. Relação entre o Tempo de Uso e Qualidade de Vida em Usuários de Prótese Total no Estado do Amapá. BJIHS. 2019;1(6):152-168.

21. Oliveira B S, Delgado S E, Brescovici S M. Alterações das funções de mastigação e deglutição no processo de alimentação de idosos institucionalizados. Rev. Bras. Geriatr. Gerontol. 2014; 17(3):575-558

CORRESPONDÊNCIA

Cátia Maria Guerra Fonseca

Av. Prof. Moraes Rego, 1235, Cidade Universitária

Recife - PE, Brazil, 50670-901

Email: catiamfguerra@gmail.com 